\title{
ROAD TO QUANTUM BOXES
}

\author{
L.L. Chang \\ Hong Kong University of Science and Technology \\ Clear Water Bay, Kowloon, Hong Kong
}

\begin{abstract}
Quantum wells and superlattices have evolved over the last two decades to become a prominent multi-disciplinary field in condensed matter science. From the first observation of resonant tunneling to the recent realization of the quantum box, in this work we provide a summary description of the historical development of the field, known today as quantum structures. The focus is on the physics aspect with emphasis on important experimental observations that collectively define the field and set its direction of development.
\end{abstract}

PACS numbers: 73.20.Dx, 73.61.-r, 78.66.-w

It has been more than two decades since the superlattices and quantum wells were first proposed and realized. This achievement has dramatically changed the landscape of semiconductor research. Indeed, it has opened up a new field in which materials, physics and electronics are pursued in artificially synthesized structures on the scale of atomic dimensions [1]. Quantum states are created with prescribed configurations, leading to unprecedented transport, optical and magnetic properties. We show in Table I the multidisciplinary nature of the field, ranging from the preparation and characterization of the structures to the exploration and investigation of new physical phenomena and device applications. Over the years, the field has experienced tremendous expansion and growth and has developed to become a dominant theme in condensed matter science, known today as quantum structures or artificially structured materials or, loosely, nanostructures.

The major focus of our interest in quantum structures has been on their physics aspects. This focus will remain in this work as we attempt to look back and give a brief account of its historical development. In Table II some of the important events are shown in chronological order with emphasis on experimental observations. The list is no doubt incomplete in view of the vast, worldwide effort in this field of research and the very large number of reports in the literature. In this table a few items in the development of electronic and optoelectronic devices are also included.

In the early 1970's, amid an environment of skepticism, we started our experimental effort to fabricate ultrathin semiconductor layers in pursuance of the quantum size effect. Guided by the theoretical prediction of a negative-resistance [2], 
TABLE I

Multidisciplinary studies in quantum structures.

\begin{tabular}{l|l|l|l}
\hline $\begin{array}{c}\text { Material } \\
\text { Synthesis }\end{array}$ & $\begin{array}{l}\text { Structure } \\
\text { Characterization }\end{array}$ & $\begin{array}{l}\text { Semiconductor } \\
\text { Physics }\end{array}$ & \multicolumn{1}{|c}{$\begin{array}{c}\text { Electronic } \\
\text { Device }\end{array}$} \\
\hline \hline MBE & $\begin{array}{l}\text { X-Ray } \\
\text { Diffraction }\end{array}$ & $\begin{array}{l}\text { Energy } \\
\text { Quantization }\end{array}$ & $\begin{array}{l}\text { Heterojunction } \\
\text { FET }\end{array}$ \\
\hline MOCVD & $\begin{array}{l}\text { Electron } \\
\text { Microscopy }\end{array}$ & $\begin{array}{l}\text { Electron } \\
\text { Dimensionality }\end{array}$ & $\begin{array}{l}\text { Heterojunction } \\
\text { Bipolar }\end{array}$ \\
\hline $\begin{array}{l}\text { Liquid } \\
\text { Phase }\end{array}$ & Electron & Magnetic & Resonant \\
Diffraction & Quantization & Tunneling Device \\
\hline Sputtering & Auger & Lattice & Hot Electron \\
& Spectroscopy & Vibration & Device \\
\hline Solid & Secondary Ion & Collective & Quantum \\
Phase & Microscopy & Excitation & Well Laser \\
\hline Ion Beam & Nuclear & Localization & Intraband \\
& Backscattering & & Infrared Detector \\
\hline Patterned & X-Ray & Electron & Optical \\
Deposition & Reflection & Dynamics & Bistability \\
\hline Self & EXAFS & Quantum & Quantum \\
Assembly & & Wires \& Dots & Interference Device \\
\hline STM & STM & Single Electron & Single Electron \\
& & Efrect & Transistor
\end{tabular}

initial work was focussed on transport properties in the direction perpendicular to the layer plane, and the results were interpreted by electron deceleration in the superlattice subband [3]. But a clear demonstration of the formation of quantum states had to wait for the observation of resonant tunneling in double barrier [4] and superlattice structures [5]. Inherent interest in the tunneling process led subsequently to extensive studies of the coherent versus incoherent nature of the process itself [6] and the dynamic behaviour of the charge built-up [7]. More recently, resonant tunneling was also applied to structures under magnetic fields both to observe electron transport through Landau levels $[8]$ and to deduce dispersion relations in the band structure [9]. Tunneling in zero-dimensional systems was also pursued when such systems became realized later [10]. Indeed, resonant tunneling has developed to be a large field in its own right in parallel with the development of quantum structures.

The successful demonstration of quantum states in man-made layers aroused great interest, leading to their investigations with different experiments. One important set of experiments involved optical radiation, including absorption, luminescence and Raman scattering [11-13]. Being spectroscopic in nature, optical 
TABLE II

Events in the development of quantum structures.

\begin{tabular}{l|l}
\hline \hline Year & \multicolumn{1}{|c}{ Events } \\
\hline $1969-70$ & Superlattice Proposal \\
$1972-73$ & Formation of Superlattice \\
1974 & Resonant Tunneling \\
1974 & Subband Transport \\
$1974-75$ & Optical Transition \\
1976 & Atomically Smooth Interface \\
$1977-79$ & Quantum Well Laser \\
1977 & Electron Dimensionality \\
$1977-79$ & Intersubband \& Phonon Interactions \\
1978 & Modulation Doping \\
$1978-79$ & Semimetallic Superlattice \\
1980 & Phonon Superlattice \\
$1980-81$ & Integral Quantum Hall Effect \\
1981 & Heterojuction Field-Effect Transistor \\
1982 & Fractional Quantum Hall Effect \\
1982 & Optical Bistability \\
1982 & Strained Superlattice \\
1983 & Microwave Resonant Tunneling Diode \\
1984 & Diluted Magnetic Superlattice \\
$1986-87$ & One-Dimensional Electron System \\
1987 & Intraband Infrared Detector \\
1988 & Point Contact Conductance \\
1988 & Zero-Dimensional Electron System \\
1988 & Stark Ladder \\
$1988-89$ & Reentrant Insulating Phase \\
$1989-90$ & Coulomb Blockade \\
1990 & SiGe Bipolar Transistor \\
1991 & ZnSe Blue Laser \\
$1992-93$ & Bloch Oscillations \\
1994 & Cascade Infrared Laser \\
&
\end{tabular}

measurements provide direct information on subband energies, excitonic processes and collective excitations [14]. The wave functions of the excitons are severely restricted by the heterostructure potential, resulting in a significant increase in their binding energies, as measured from the luminescence spectra [15]. Similar experiments were also used to study the enhancement of the binding energies of impuri- 
ties which also involve Coulombic interactions, and the effect of band mixing and crossing under an electric field [16]. In Raman scattering, on the other hand, by registering the frequency shift of incident and scattered photons, information can be obtained about elementary excitations such as phonons and plasmons as well as interband transitions and their resonant behaviour. New phonon modes were also observed, including both confined and propagating modes [17,18], leading to the notion of the superlattice of phonons in analogy to that of electrons.

In addition to optics, experiments under the application of a magnetic field were initiated, following the observation of quantum states in resonant tunneling. The field quantizes the electron motion in the perpendicular plane, and this additional quantization makes it particularly suitable for use in studying low-dimensional systems. Oscillations in magneto-resistance were observed as functions or magnitude and orientation of the field, demonstrating two-dimensionality in an isolated quantum well and variable two- to three-dimensionality in a superlattice [19]. Studies that followed extended rapidly to magneto-absorption and cyclotron resonance $[20,21]$, and subsequently to interactions with phonons and plasmons $[22,23]$. In a separate development, spin splittings were extensively investigated, including the enhancement and oscillations of the $g$-factor in a magnetic field as a result of self-energy exchange interaction [24]. Another topic drawing broad interest is related to the scattering process under high fields and the resulting density-of-states and Landau-level broadening.

By the time the decade of the 1970's was drawing to a close, most of the key features of a quantum structure for it to be viewed as a new material had been demonstrated. Each phenomenon in the structure influences a specific physical parameter which characterizes and governs the properties of the material: electric quantization creates new schemes of subband energies; low-dimensionality of the electron system leads to different density-of-states functions; exciton and impurity confinements produce enhanced bindings; and modified lattice vibrations result in new phonon modes. Added to these features was the invention of modulation doping to reduce impurity scattering by spatially separating impurities in the barriers from carriers in the wells $[25,26]$. Since all these features can be tailored by designing the quantum structure in terms of its composition, thickness and configuration, the quantum structure represents a new class of engineered electronic materials.

With the improvement in quality and availability of samples, research activities in quantum structures grew exponentially both in scope and in depth in the decade of the 1980's. Many of the earlier observations were further pursued, as described above, which led to better understanding of the observations and to broadened research in related areas. Furthermore, quantum structures of different semiconductors, no longer limited to GaAs-GaAlAs which had been the predominant system of investigation from the beginning, began to come to the scene and became widely pursued. This success widened the field in several different directions of development. The first was the effect of band structure; arising from the use of various combinations of semiconductors. Notable examples were InAs-Gasb [27], $\mathrm{HgTe}-\mathrm{CdTe}$ [28] and $\mathrm{Si}-\mathrm{SiGe}$ [29, 30]. The broken-gap situation in the case of InAs-Gasb was of particular interest for it gave rise to the spatial 
separation of carriers and the formation of semimetallic structure $[31,32]$. The second development is related to the effect of strain, a consequence of mismatch of lattice constants from different materials [33]. This opened up the field to a large variety of combinations of semiconductors, and also made it possible to take advantage of the influence of strain on the band structure to produce interesting phenomena [34]. The third development had to do with the introduction of special materials to the field of quantum structures, such as the semimagnetic or diluted magnetic semiconductor [35]. The magnetic exchange interaction resulted in significant observations of dimensional crossover of phase transitions and spin polarization and relaxation dynamics $[36,37]$.

Coinciding with the hectic pace in the 1980's for various investigations of physical phenomena in different material systems, two specific observations stood out: the quantum Hall effect under magnetic fields near the beginning of the decade [38-40] and the Stark ladder formation under electric fields near the end $[41,42]$. While the integral quantum Hall effect can be largely understood on the basis of localization, the fractional quantum Hall effect is a many-body effect, involving quasi-particle excitations [43]. The richness in physics continued to produce unexpected results and new insight into the problem, including edge states, hierarchical structure, spin unpolarized states [44,45] and multilayer quantum liquids $[46,47]$. The possibility of a Wigner transition has always drawn great attention [48-50], and so has been the advancement in theory such as the most recent formulation of composite fermion to provide a unified explanation to both the integral and fractional effects $[51,52]$. In the other development, electric fields had long been used to alter the quantum states and polarized the electron-hole distribution [53]. As the field increases, the extended states in a superlattice tend to be localized, leading to the formation of the Stark ladder. The observation of the ladder shed new light on the fundamental question of the Bloch oscillation and inspired extensive experimental efforts. By use of different techniques of measurement, various manifestations of this effect were reported, including transport [54], four-wave mixing [55] and coherent electromagnetic radiation [56].

During the course of studies of quantum wells and superlattices, which are essentially two-dimensional electron systems with potential confinement in one direction, the possibility of providing additional confinement in the other two directions had constantly intrigued people working in the field. That the staircase density-of-states in two-dimensionality becomes spiked and delta-function as the dimensionality is further reduced to one and zero would undoubtedly offer new possibilities in pursuance of physics. Various clever schemes and processing techniques were attempted to produce such structures with different degrees of success: electron-beam and X-ray lithography, focussed ion-beam deposition, scanning tunneling microscopy [57], various chemical processes and special growth techniques on edges, vicinal surfaces and nonplanar substrates $[58,59]$. The most commonly used structure today is fabricated laterally on the plane of a two-dimensional electron gas by electron-beam patterning, reactive-ion etching and proper gating with applied voltages. One-dimensional quantum wire and zero-dimensional quantum dots or boxes are produced by use of such patterning in one and two directions, respectively. 
Evidence for the formation of quantum wires were provided from both transport [60,61], optical [62] and capacitance measurements [63]. The last experiment probes directly the density-of-states, showing well-defined oscillations as the Fermi level crosses the one-dimensional quantum states. Similar capacitance measurements as well as resonant tunneling measurements were subsequently applied to quantum boxes to demonstrate the zero-dimensional quantum states $[10,63]$. The success brought about a new vigor and enthusiasm to the research of quantum structures and, by applying the knowledge and techniques acquired earlier from the two-dimensional studies to these lower-dimensional systems, led rapidly to interesting results in transport and optical properties under both electric and magnetic fields. One observation of particular importance was that of point-contact, quantized conductance under a narrow constriction [64]. The conductance showed steps and plateaux arising from successive population of the one-dimensional channels. Ballistic transport was brought under focus. The two fields in small structures began to converge: quantum wells and superlattices which traditionally emphasize quantization, and mesoscopic systems which are concerned with phase coherence. Another observation of significant impact was that of Coulomb blockade, as manifested in the conductance of quasi-isolated electron dots as a result of charge quantization [65-67]. This illustrated clearly the exciting opportunity of dealing with a system containing only few electrons or, more generally, other types of particles such as impurities and spins.

Riding on the momentum of small structures, studies in the field of quantum structures showed no signs of abatement as it entered the decade of 1990's. We name here just a few notable examples: the dynamic localization and multiphoton emission under terahertz fields in superlattices [68], the enhancement of effective mass of composite fermions near half-filled Landau levels in the fractional quantum Hall regime [69], the effect of electron-electron interaction in transport through quantum wires in the Luttinger liquid $[70,71]$ and single electron ccupation [72] and tunneling [73] in quantum dots. Added to these are the voluminous output which continued in the more tradition studies in the two-dimensional electron systems. We show in Fig. 1 the growth of the field in terms of the number of papers related to the field presented in the last two decades at the International Conference on the Physics of Semiconductors, which started in 1950 and has been since held biennially. Clearly seen are the incubation period in the 1970's, the steep rise in the 1980's and the continued growth on the logarithmic scale in the 1990's when its ratio to the total number of papers presented at the Conference reached beyond 50 percent.

In writing this summary account of the development of quantum structures, from the first observation of resonant tunneling to the success of achieving quantum boxes, we are amazed at the number of publications and the number of research people in this field. The field has enjoyed an uninterrupted growth, highlighted by major observations and breakthroughs which invariably carried the frontiers of research to subsequent plateaux. At present, on-going activities contain sufficient new and exciting features to push the research forward, pending further discoveries which are unpredictable by their very nature. It should be mentioned in this connection that great progress has been made recently in clusters and tubes, 


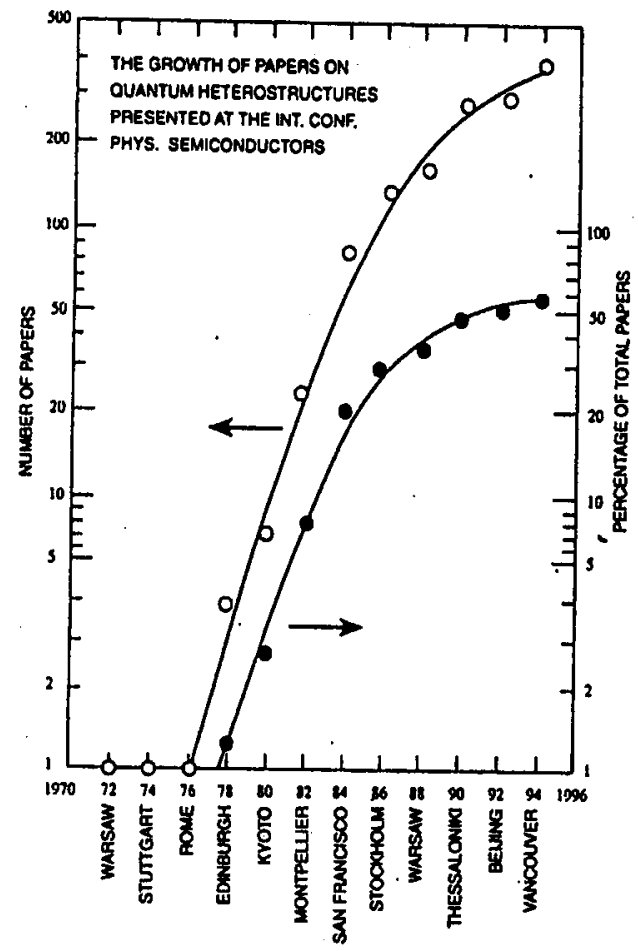

Fig. 1. Papers on quantum structures presented at the International Conference on the Physics of Semiconductors to illustrate the growth of research in this field.

embedded particles and controlled nanocrystals $[74,75]$, offering the possibility of making three-dimensional molecules and superlattices. They may conceivably provide the next frontier in quantum-box research.

\section{References}

[1] See, zor a review, L.L. Chang, in: Highlights in Condensed Matter Physics and Future Prospects, Ed. L. Esaki, Plenum, New York 1991, p. 83.

[2] L. Esaki, R. Tsu, IBM J. Res. Develop. 14, 61 (1970).

[3] L.L. Chang, L. Esaki, W.E. Howard, R. Ludeke, J. Vac. Sci. Technol. 10, 11 (1973).

[4] L.L. Chang, L. Esaki, R. Tsu, Appl. Phys. Lett. 24, 593 (1974).

[5] L. Esaki, L.L. Chang, Phys. Rev. Lett. 33, 495 (1974).

[6] S. Luryi, Appl. Phys. Lett. 47, 490 (1985).

[7] V.J. Goldman, D.C. Tsui, J.E. Cunningham, Phys. Rev. Lett. 58, 1256 (1987).

[8] E.E. Mendez, L. Esaki, W.I. Wang, Phys. Rev. B 33, 2893 (1986).

[9] M.L. Leadbeater, L. Eaves, P.E. Simmons, G.A. Toombs, F.W. Sheard, P.A. Claxton, G. Hill, M.A. Pate, Solid State Electron. 31, 707 (1988).

[10] M.S. Reed, J.N. Randall, R.J. Aggarwal, R.J. Matyi, T.M. Moore, A.E. Wetsel, Phys. Rev. Lett. 60, 535 (1988). 
[11] R. Dingle, W. Wiegmann, C.H. Henry, Phys. Rev. Lett 33, 827 (1974).

[12] R.C. Miller, W.T. Tsang, W.A. Nordland, Phys. Rev. B 21, 1569 (1980).

[13] P. Manuel, G.A. Sai-Halasz, L.L. Chang, C.A. Chang, L. Esaki, Phys. Rev. Lett. 37, 1701 (1976).

[14] A. Pinczuk, H.L. Stormer, R. Dingle, J.M. Worlock, W. Wiegmann, A.C. Gossard, Solid State Commun. 32, 1001 (1979).

[15] R.C. Miller, D.A. Kleinman, W.T. Tsang, A.C. Gossard, Phys. Rev. B 24, 1134 (1981).

[16] R.T. Collins, L. Vina, W.I. Wang, L.L. Chang, L. Esaki, K. von Klitzing, K. Ploog, Phys. Rev. B 36, 1531 (1987).

[17] C. Covard, R. Merlin, M.V. Klein, A.C. Gossard, Phys. Rev. Lett. 45, 298 (1980).

[18] B. Jusserand, D. Paque, A. Regrency, Phys. Rev. B 30,6245 (1984).

[19] L.L. Chang, H. Sakaki, C.A. Chang, L. Esaki, Phys. Rev. Lett. 25, 1489 (1977).

[20] H.L. Stormer, R. Dingle, A.C. Gossard, W. Wiegmann, M.D. Sturge, Solid State Commun. 29, 705 (1979).

[21] H.J.A. Bluyssen, J.C. Maan, P. Wyder, L.L. Chang, L. Esaki, Solid State Commun. 31, 35 (1979).

[22] D.C. Tsui, Th. Englert, A.Y. Cho, A.C. Gossard, Phys. Rev. Lett. 44, 341 (1980).

[23] D. Olego, A. Pinczuk, A.C. Gossard, W. Wiegmann, Phys. Rev. B 25, 7867 (1982).

[24] Th. Englert, D.C. Tsui, A.C. Gossard, C. Uihlein, Surf. Sci. 113, 295 (1982).

[25] R. Dinlge, H.L. Stormers, A.C. Gossard, W. Wiegmann, Appl. Phys. Lett. 33, 665 (1978).

[26] L.C. Witkowski, T.J. Drummond, C.M. Stanchak, H. Morkoc, Appl. Phys. Lett. 37, 1033 (1980).

[27] H. Sakaki, L.L. Chang, R. Ludeke, C.A. Chang, G.A. Sai-IIalasz, L. Esaki, Appl. Phys. Lett. 31, 211 (1977).

[28] J.P. Faurie, A. Million, J. Paiguet, Appl. Phys. Lett. 41, 713 (1982).

[29] E. Kasper, H.J. Horzog, H. Kibbel, Appl. Phys. 8, 199 (1975).

[30] H.M. Manasevit, I.S. Gergis, A.B. Jones, Appl. Phys. Letl. 41, 464. (1982).

[31] L.L. Chang, N.J. Kawai, G.A. Sai-Halasz, R. Ludeke, L. Esaki, Appl. Phys. Lett. 35, 939 (1979).

[32] L.L. Chang, G.A. Sai-Halasz, L. Esaki, R.L. Aggarwal, J. Vac. Sci. Technol. 19, 589 (1981).

[33] P.L. Gourley, R.M. Biefield, J. Vac. Sci. Technol. 21, 473 (1982).

[34] P. Voisin, C. Delalande, M. Voos, L.L. Chang, A. Segmuller, C.A. Chang, L. Esaki, Phys. Rev. B 30, 2276 (1984).

[35] R.N. Bicknell, R.K. Yanka, N.C. Giles, D.K. Blanks, E.L. Buckland, J.F. Schetzina, Appl. Phys. Lett. 45, 92 (1984).

[36] D.D. Awschalom, J.M. Hong, L.L. Chang, G. Grinstein, Phys. Rev. Lett. 59, 1733 (1987).

[37] M.R. Freeman, D.D. Awschalom, J.M. Hong, L.L. Chang, Phys. Rev. Lett. 64, 2430 (1990).

[38] K. Von Klitzing, G. Dorda, M. Pepper, Phys. Rev. Lelt. 45, 494 (1980). 
[39] D.C. Tsui, A.C. Gossard, Appl. Phys. Lett. 38, 550 (1981).

[40] D.C. Tsui, H.L. Stormer, A.C. Gossard, Phys. Rev. Lett. 48, 1559. (1982).

[41] E.E. Mendez, F. Agullo-Rueda, J.M. Hong, Phys. Rev. Lett. 60, 2426 (1988).

[42] P. Voisin, J. Bleuse, C. Bouche, S. Gaillard, C. Alibert, A. Regreny, Phys, Rev. Lett. 61, 1639 (1988).

[43] R.B. Laughlin, Phys. Rev. Lett. 50, 1395 (1983).

[44] R.G. Clark, R.J. Nicholas, A. Usher, C.T. Foxon, J.J. Harris, Surf. Sci. 170, 141 (1986).

[45] R. Willett, J.P. Eisenstein, H.L. Stormer, D.C. Tsui, A.C. Gossard, J.H. English, Phys. Rev. Lelt. 59, 1776 (1987).

[46] Y.W. Suen, L.W. Engel, M.B. Santos, M. Shayegan, D.C. Tṣui, Phys. Rev. Lett. 68, 1379 (1992).

[47] J.P. Eisenstein, G.S. Boebinger, L.N. Pfeiffer, K. West, S. He, Phys. Rev. Lett. 68, 1383 (1992).

[48] V.J. Goldman, M. Shayegan, D.C. Tsui, Phys. Rev. Lett. 61, 881 (1988).

[49] R.L. Willett, H.L. Stormer, D.C. Tsui, L.N. Pfeiffer, K.W. West, B. Baldwin, Phys. Rev. B 38, 7881 (1989).

[50] V.J. Goldman, M. Santos, M. Shayegan, J. Cunningham, Phys. Rev. Lett. 65, 2189 (1990).

[51] J.K. Jain, Phys. Rev. Lett. 63, 199 (1989).

[52] B.I. Halperin, P.A. Lee, N. Reed, Phys. Rev. B 47, 7312 (1993).

[53] E.E. Mendez, G. Bastard, L.L. Chang, L. Esaki, H. Morkoc, R. Fischer, Phys. Rev. $B$ 26, 7101 (1982).

[54] A. Sibille, J.F. Palmer, F. Mollot, Appl. Phys. Lett. 60, 547 (1992).

[55] K. Leo, P.H. Boliva, F. Bruggemann, R. Schwedler, K. Koehler, Solid State Commun. 84, 943 (1992).

[56] C. Waschke, H.G. Roskos, R. Schwedler, K. Leo, H. Kurz, K. Koehler, Phys. Rev. Lett. 70, 3319 (1993).

[57] D.M. Eigler, E.K. Schweizer, Nature 344, 524 (1990).

[58] P.M. Petroff, A.C. Gossard, W. Wiegmann, Appl. Phys. Lett. 45, 620 (1984).

[59] A.C. Warren, D.A. Antoniades, H.L. Smith, Phys. Rev. Lett. 56, 1858 (1986).

[60] K.F. Berggren, T.J. Thorton, D.J. Newton, M. Pepper, Phys. Rev. Lett. 57, 1769 (1986).

[61] W. Hansen, M. Horst, J.P. Kotthaus, V. Merkt, C. Sikorski, K. Ploog, Phys. Rev. Lett. 58, 2586 (1987).

[62] T.P. Smith, H. Arnot, J.H. Hong, C.M. Knoedler, S.E. Laux, H. Schmid, Phys. Rev. Lett. 59, 2802 (1987).

[63] T.P. Smith, K.Y. Lee, C.M. Knoedler, J.M. Hong, D.D. Kern, Phys. Rev. $B$ 38, 2172 (1988).

[64] B.J. van Wees, H. van Houton, C.W.J. Beenakker, J.G. Williamson, L.P. Kouwenhoven, D. van der Marel, C.T. Foxon, Phys. Rev. Lett. 60, 648 (1988).

[65] T.A. Fulton, G.J. Dolan, Phys. Rev. Lett. 59, 109 (1987).

[66] D. Delsing, K.K. Likharev, L.S. Kuzmin, T: Claeson, Phys. Rev. Lett. 63, 1861 (1989). 
[67] V. Meirav, M.A. Kastner, S.J. Wind, Phys. Rev. Lett. 65, 771 (1990).

[68] B.J. Keay, S. Zeuner, S.J. Allen, K.D. Maranowski, A.C. Gossard, U. Bhattacharya, M.J.W. Rodwell, Phys. Rev. Lett. 75, 4102 (1995).

[69] R.R. Du, H.L. Stormer, D.C. Tsui, A.S. Yeh, L.N. Pfeiffer, K.W. West, Phys. Rev. Lett. 73, 3274 (1994).

[70] S. Tarucha, T. Honda, T. Saku, Solid State Commun. 94, 413 (1995).

[71] D.L. Maslov, Phys. Rev. B 52, 14368 (1995).

[72] B. Meurer, D. Heitmann, K. Ploog, Phys. Rev. Lett. 68, 1371 (1992).

[73] R.C. Ashoori, H.L. Stormer, J.S. Weiner, L.N. Pfieffer, S.J. Pearton, K.W. Baldwin, K.W. West, Phys. Rev. Lett. 68, 3088 (1992).

[74] C.B. Murray, D.J. Norris, M.G. Bawerdi, J. Am. Chem. Soc. 115, 8706 (1993).

[75] J.E. Bowen Katari, V.L. Colvin, A.P. Alvisatos, J. Phys. Chem. 98, 4109 (1994). 